\title{
CHRONIC DAILY HEADACHE IN YOUNG ADOLESCENTS
}

Investigators at Taipei Veterans General Hospital, Taiwan, studied the incidence and risk factors of chronic daily headache $(\mathrm{CDH})$ in a field cohort of 3342 adolescents aged 13 to 14 in 3 middle schools, from 2005 to 2007. Questionnaires included the Adolescent Depression Inventory and Pediatric Migraine Disability Assessment. Sixtythree subjects (21 boys/42 girls) developed $\mathrm{CDH}$ with an incidence rate of 1.13 per 100 PYs; 37 had chronic migraine [CM] (0.66 per 100 PYs) and 22 had chronic tension-type headache [CTTH] (0.39 per 100 PYs). Thirty-three subjects $(52 \%)$ had a baseline diagnosis of migraine.

Independent risk factors for $\mathrm{CDH}$ included female gender, acute family financial distress, obesity, higher headache frequency, and a baseline diagnosis of migraine. A higher headache frequency was the only identical risk factor for $\mathrm{CDH}, \mathrm{CM}$, and CTTH. A baseline diagnosis of migraine and obesity were significant predictors for both $\mathrm{CM}$ and $\mathrm{CDH}$. Female gender was a significant predictor for both CTTH and CDH. (Lu S-R, Fuh J-L, Wang S-J, et al. Incident and risk fctors of chronic daily headache in young adolescents: A school cohort study. Pediatrics 2013 Jul;132(1):e9-e16). (Response: Shuu-Jiun Wang MD. E-mail: sjwang@vghtpe.gov.tw).

COMMENT. Treatment of acute migraine. Evidence for the pharmacological treatment of acute migraine in children and adolescents is poor, according to a review by Wober-Bingol C. Paediatr Drugs 2013 Jun;15(3):235-46. Only a few randomized controlled studies are published and high placebo rates are the rule. Acetaminophen (paracetamol) and ibuprofen are accepted as drugs of first choice, but the evidence is poor for acetaminophen and limited for ibuprofen. Among 14 studies on triptans in adolescents, 9 showed some superiority over placebo, and among 6 studies in children, 5 suggested some superiority over placebo. Sumatriptan nasal spray and zolmitriptan nasal spray are approved for adolescent use in Europe; almotriptan is approved for adolescents in the USA, and rizatriptan for patients aged 6-17 years. A combination of sumatriptan and naproxen is found effective in one study in adolescents.

\section{HEADACHE AND CALL-FLEMING SYNDROME}

Investigators at centers in New Jersey report a 16-year-old girl with a history of anxiety, ADHD, and migraine, treated with several medications and presenting with 2 weeks of worsening headaches. CT was normal, but MRI angiography revealed cortical subarachnoid hemorrhage. CT angiography and follow-up digital subtraction angiography confirmed extensive vasoconstriction of small- to medium-sized cerebral arteries. Sertraline and methylphenidate were discontinued, and nifedipine (a calcium channel blocker) was introduced. Symptoms rapidly improved, and repeat CT angiography at 2 months showed no vasoconstriction. Call-Fleming syndrome, usually called "reversible cerebral vasoconstriction syndrome," is an important cause of thunderclap headache in adults and should be considered in the pediatric population presenting with severe headache. (Bain J, Segal D, Amin R, Monoky D, Thompson SJ. Call-Fleming syndrome: Headache in a 16-year-old girl. Pediatr Neurol 2013 
Aug;49(2):130-133.e1). (Response: Dr Bain, UMDNJ - New Jersey Medical School; Pediatrics: 185 S Orange Ave, Newark, NJ 07101. E-mail: jenniferbain08@gmail.com).

COMMENT. Reversible cerebral vasoconstriction syndrome, sometimes called Call-Fleming syndrome (Call GK, et al. Stroke 1988 Sep;19(9):1159-70) is rare in children but should be considered in the differential diagnosis of a child with a history of migraine and presentation with thunderclap headache. Many risk factors in addition to migraine are listed, including vasoactive drugs (triptans, amphetamines, ginseng, nicotine patch, oral contraceptives, diet pills, selective serotonin reuptake inhibitors), blood products, and trauma. The disorder is self-limiting, the headache resolving in 3 weeks and vasoconstriction resolving by 12 weeks. The majority has no residual symptoms and no recurrence of headaches, although stroke is a potential complication. Children treated with methylphenidate or amphetamines for ADHD and having a history of headache should be followed closely and the stimulant discontinued if headaches are persistent.

\section{DEVELOPMENTAL DISORDERS}

\section{CORPUS CALLOSUM IN SMITH-LEMLI-OPITZ SYNDROME}

Investigators at the Kennedy Krieger Institute and Johns Hopkins University, Baltimore; and the National Institutes of Health, Bethesda, MD, studied the relation between the size of the corpus callosum (CC), level of serum 7-dehydrocholesterol (7DHC), and severity of developmental delay in 36 subjects with Smith-Lemli-Opitz syndrome (SLOS), compared to 36 normal controls. The mean age was 3.9 yrs (range 0.2 - $12.5 \mathrm{yrs}$ ); 18 boys and 18 girls. Shorter CC length measured on one midsagittal image and smaller $\mathrm{CC}$ area correlated with a lower developmental quotient in gross motor and language domains, ranging from mild to severe, but not with fine motor or adaptive skills. Also, CC length and area negatively correlated with the 7DHC and 8DHC levels, and positively correlated with serum total cholesterol level. (Lee RW, Yoshida S, Jung ES, Mori S, Baker EH, Porter FD. Corpus callosum measurements correlate with developmental delay in Smith-Lemli-Opitz syndrome. Pediatr Neurol 2013 Aug;49(2):107-12). (Response: Dr Lee, Kennedy Krieger Institute, Baltimore, MD 21205. E-mail: leer@kennedykrieger.org).

COMMENT. SLOS (or 7-dehydrocholesterol reductase deficiency) is an autosomal recessive metabolic and developmental congenital disorder, first described in 1964 (Smith DW, Lemli L, Opitz JM. A newly recognized syndrome of multiple congenital anomalies. J Pediatr 1964 Feb;64:210-7). The most commonly observed features include dysmorphic faces, microcephaly, 2-3 syndactyly of toes, polydactyly, growth retardation, intellectual disability, cleft palate, and hypospadias. Hypoplasia/agenesis of the corpus callosum is reported less frequently, as well as holoprosencephaly. Mutations in the DHCR7 gene are the cause, the enzyme responsible for production of cholesterol, an essential nutrient for embryonic development. Sequencing analysis of DHCR7 detects $\sim 96 \%$ of known mutations. (Irons M. SmithLemli-Opitz Syndrome. In: Pagon RA, Adam MP, Bird TD, et al., editors. GeneReviews [Internet]. Seattle (WA): University of Washington, Seattle; 1993-2013). 